The Astrophysical. Journal, 472: L97-L100, 1996 December 1

1096. The American Astronomical Socicty. All rights reserved. Printed in U.S.A.

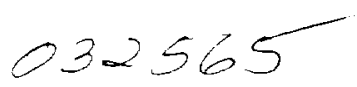

\title{
AN ULTRAVIOLET-EXCESS OPTICAL CANDIDATE FOR THE LUMINOUS GLOBULAR CLUSTER X-RAY SOURCE IN NGC 1851'
}

\author{
Eric W. Deutsch, Scott F. Anderson, and Bruce Margon \\ Department of Astronomy, University of Washington, Box 351580, Seattle, WA 98195-1580; deutsch (castro.washington.edu; \\ anderson $(a$ astro.washington.edu; margon $a$ ast ro. washington.edu \\ AND \\ Ronald A. DOWNES \\ Space Telescope Science Institute, 3700) San Martin Drive, Baltimore, MD 21218; downes(a stsci.edu \\ Received 1996 Alugust 1; accepted 1996 Scptember 19
}

\begin{abstract}
The intense, bursting X-ray source in the globular cluster NGC 1851 was one of the first cluster sources discovered, but has remained optically unidentified for 25 years. We report here on results from Hubble Space Telescope WFPC2 multicolor images in NGC 1851. Our high spatial resolution images resolve $\sim 200$ objects in the $3^{\prime \prime}$ radius Einstein X-ray error circle, 40 times as many as in previous ground-based work. A color-magnitude diagram of the cluster clearly reveals a markedly UV-excess object with $B \sim 21,(U-B) \sim-0.9$, only $2^{\prime \prime}$ from the X-ray position.

The UV-excess candidate is $0 . " 12$ distant from a second, unremarkable star that is 0.5 mag brighter in $B$; thus ground-based studies of this field are probably impractical. Three other UV-excess objects are also present among the $\sim 16,000$ objects in the surveyed region of the cluster, leaving a $\sim 5 \%$ probability that a UV-excess object has fallen in the X-ray error circle by chance. No variability of the candidate is seen in these data, although a more complete study is required. If this object is in fact the counterpart of the $\mathrm{X}$-ray source, previous inferences that some globular cluster X-ray sources are optically subluminous with respect to low-mass X-ray binaries in the field are now strengthened.
\end{abstract}

Subject headings: globular clusters: individual (NGC 1851) - stars: neutron - ultraviolet: stars $\mathrm{X}$-rays: bursts $-\mathrm{X}$-rays: stars

\section{INTRODUCTION}

It has been known for over two decades that cluster X-ray sources are overabundant with respect to those in the field (Katz 1975; Clark 1975). While globular clusters only contain $10^{-4}$ of the mass of the Galaxy, over $10^{2}$ of the bright X-ray sources $\left(L_{\mathrm{X}} \geq 10^{36} \mathrm{ergs} \mathrm{s}^{-1}\right)$ are found in globulars. The cause of this discrepancy may be a difference in the nature of globular cluster X-ray sources from those in the field or more likely an enhancement of their formation probability.

Despite a quarter-century of effort, there are very few intense globular cluster X-ray sources with convincingly identified optical counterparts. AC 211 in M15 (Aurière, Le Fèvre, \& Terzan 1984; Charles, Jones, \& Naylor 1986) was discovered over a decade ago; it is surprisingly optically luminous. A variety of candidates in NGC 6712 were offered (e.g., Cudworth 1988; Nieto et al. 1990; Bailyn et al. 1991; Aurière \& Koch-Miramond 1992) and the counterpart, star S, was eventually unambiguously confirmed by Anderson et al. (1993) with $H S T$ images. A counterpart for the X-ray source in NGC 6624 was discovered to dominate the cluster light at $1400 \AA$ in HST Faint Object Camera images by King et al. (1993). These three optical counterparts discovered so far show a wide range in optical luminosity, and recently the spectra of $\mathrm{AC} 211$ and star S were found to differ significantly (Downes et al. 1996).

The scarcity of optical counterparts is almost certainly

\footnotetext{
1 Based on observations with the NASA/ESA Hubble Space Telescope obtained at the Space Telescope Science Institute, which is operated by the Association of Universities for Research in Astronomy. Inc., under NASA contract NAS5-26555.
}

primarily due to the extreme crowding in the clusters, which limits the utility of ground-based identification programs. As part of the Hubble Space Telescope Faint Object Spectrograph team's observing program, we have obtained multicolor $H S T$ images of several globular clusters containing $\mathrm{X}$-ray sources, with the aim of resolving optical counterparts in crowded fields and studying their spectra. We report here on the results from WFPC2 images in NGC 1851.

$X$-ray observations of the strong source in NGC 1851 are virtually as old as satellite X-ray astronomy, dating back to the days of OSO 7 (Clark, Markert, \& Li 1975); Forman \& Jones (1976) first observed the bursting nature of X0512-401 with Uhuru. Recent X-ray observations of the system have been made and reviewed by Callanan, Penny, \& Charles (1995). This source would seem a particularly good candidate for an optical identification, as there is an accurate Einstein HRI X-ray position (Grindlay et al. 1984), and it lies over 2 core radii $\left(\sim 12^{\prime \prime}\right)$ north of the cluster center.

Ground-based imaging studies in NGC 1851 by Aurière, Bonnet-Bidaud, \& Koch-Miramond (1994, hereafter ABK) do not reveal any especially good optical candidate (e.g., no object with marked UV excess) for the X-ray source X0512-401. However, these authors call attention to an object they denote as X1, as the best candidate they are able to resolve in a $3^{\prime \prime}$ error circle. As its color $(U-B \sim 0)$ is no more UV than many hot blue horizontal-branch stars in the cluster, ABK speculate that X1 may be a new sort of low-mass $\mathrm{X}$-ray binary (LMXRB), in which there is very little X-ray heating of the accretion disk.

Previous studies of NGC 1851 using pre-servicing-mission 
HST images did not detect any better candidates (Margon et al. 1992; Deutsch et al. 1994). The WF/PC images used in those studies do not go nearly as deep as the WFPC2 data used in the present work. Deutsch et al. (1994) reported several objects that were bluer than $\mathrm{X} 1$ at the detection threshold of those images, but were unable to isolate any single object unusual enough to be considered a better candidate. These earlier studies would have easily revealed counterparts as luminous as AC 211 or star S. Clearly, deeper exposures are required to look for fainter objects in NGC 1851.

\section{OBSERVATIONS AND DATA REDUCTION}

\subsection{Planetary Camera Imagery}

On 1996 April 10, we obtained HST WFPC2 images of NGC 1851. Two $900 \mathrm{~s}$ exposures through the F336W filter were taken, followed by four $300 \mathrm{~s} \mathrm{F439W}$ exposures, and finally two more $900 \mathrm{~s}$ F336W exposures; the F336W and F439W filters are similar to Johnson $U$ and $B$, respectively. The frames have been processed through the standard data reduction pipeline at STScl. Further reduction was performed with software written in interactive data language (IDL) by E. W. D. or available in the IDL Astronomy User's Library (Landsman 1993). Some photometry was performed with DoPHOT (Schechter, Mateo, \& Saha 1993).

Although the images were obtained with the fine-lock mode of HST, the point-spread functions (PSFs) clearly show clongation in the $Y$ direction in the Planetary Camera (PC) images, with major- to minor-axis ratios of about 1.25 . Furthermore, while the images show excellent registration in the $X$ direction, $Y$ offsets between exposures through the same filter are as high as 0.4 pixels.

For each filter, the set of four images is combined into one master image using software written by E. W. D. First, cosmic-ray hits are identified in each image, and those pixels are set to zero in the data and "exposure mask" arrays. The identification algorithm is similar to the one described in Saha et al. (1996), and is carefully adjusted so that the undersampled cores of bright stars with subpixel shifts between images are not mistaken for cosmic-ray events. The four images and masks are aligned to $\sim 0.01$ pixel accuracy and summed. The summed frame is then divided by the mask to obtain the final image. In this way, cosmic-ray event pixels are excluded so that the information in neighboring pixels is not contaminated during interpolation.

\subsection{Astrometry}

In order to determine the position of the X-ray source coordinates on the HST PC image, we establish a coordinate system based on the HST Guide Star Catalog (GSC) reference frame. We begin with the astrometric solution from a digitized Quick V image used to generate the HST Guide Star Catalog (Lasker et al. 1990); this image, obtained directly from STScI, contains the astrometric solution in the image header. By centroiding 29 stars in the Quick $V$ image and the corresponding objects in a short ground-based $R$-band CCD image, kindly provided by $H$. Ford, the astrometric reference frame is transferred to the CCD image with an error in the solution of 0.1 . Next, the astrometric frame is transferred from the CCD image to the PC image using a set of nine common isolated stars, with error in the solution of 0.02 , negligible compared to the first step. The final result is that coordinates may be determined on the PC image to within 0.1 in the GSC reference frame. However, there may well be some systematic offset, $\sigma \sim 0$. 5 , from frames based on other reference catalogs (Russell et al. 1990).

We calculate the weighted mean of the X-ray coordinates from reprocessed Einstein HRI data in the HRICFA database obtained through the High Energy Astrophysics Science Archive Research Center Online Service, provided by the NASA Goddard Space Flight Center. These coordinates are not significantly different from those in Grindlay et al. (1984), so we use the published coordinates in this work. The $90 \%$ confidence radius is reported by Grindlay et al. (1984) as 3".

The optical position of the $\mathrm{X}$-ray coordinates chosen by ABK, which we estimate from Figure 1 of that paper, is $\sim 1^{\prime \prime}$ northeast of our location, which is not unreasonable given the different source of astrometric reference.

The left panel of Figure 1 (Plate L11) shows a $24^{\prime \prime} \times 24^{\prime \prime}$ region of the cluster through the $\mathrm{F} 336 \mathrm{~W}$ filter. A $3^{\prime \prime}$ radius error circle is overlaid, using our astrometric solution. The right panel of Figure 1 shows a $8^{\prime \prime} \times 8^{\prime \prime}$ region of the cluster through the F336W filter, centered on our optical position for the X-ray source, which we have marked with a cross.

\subsection{Photometry}

Photometry was performed on the combined F336W and F439W PC frames with the DoPHOT software (Schechter et al. 1993) and a PSF function-fitting procedure written by E. W. D. The results are similar, although DoPHOT was not able to converge accurately on some of the brighter objects; however, it performed better on the fainter objects. For this reason, we use the results derived from DoPHOT. The fitted magnitudes were calibrated with aperture photometry of several isolated objects. Aperture corrections are taken from Table $2 a$ in Holtzman et al. (1995b). The photometric measurements have not been corrected for geometric distortions in the $\mathrm{PC}$, but the simple correction for charge transfer efficiency losses detailed in Holtzman et al. (1995b) has been applied. We use the photometric zero points for the STMAG system from Table 9 in Holtzman et al. (1995a). Systematic errors for all magnitudes due to uncertainties in detector performance, absolute calibration, and filter transformations are $\sim 5 \%$.

The photometric results are displayed in Figure 2 as a color-magnitude diagram (CMD) of all $\sim 5500$ objects with reliable measurements in the $\mathrm{PC}$ frame. Objects which are mentioned in the discussion below are marked in the diagram.

\section{DISCUSSION}

While $\mathrm{ABK}$ were only able to resolve five stars within a $3^{\prime \prime}$ radius error circle, we are able to resolve and measure magnitudes for 194 objects with $15 \leq m_{439} \leq 23$.

Star X1, suggested by ABK as the best candidate, does have the brightest $m_{3,36}$ magnitude within the error circle. However, its color and magnitude are unremarkable compared with other cluster horizontal-branch stars. We resolve the star labeled as $\mathrm{X} 2$ by $\mathrm{ABK}$ into two main components, which we label $X 2 a$ and $X 2 b$. Additional, fainter stars also contribute light to $X 2$. Object $X 2 a$ is nearly in the center of our error circle, but has normal colors. In the CMD, X2b is found to have a more UV color than star $\mathrm{X} 1$, but is also unremarkable compared with other stars in the cluster (Fig. 2).

In the CMD of the entire $34^{\prime \prime} \times 34^{\prime \prime}$ PC field, there are two objects that stand out as having marked UV excess, with colors similar to known LMXRBs. One of these is 2"15 away from 


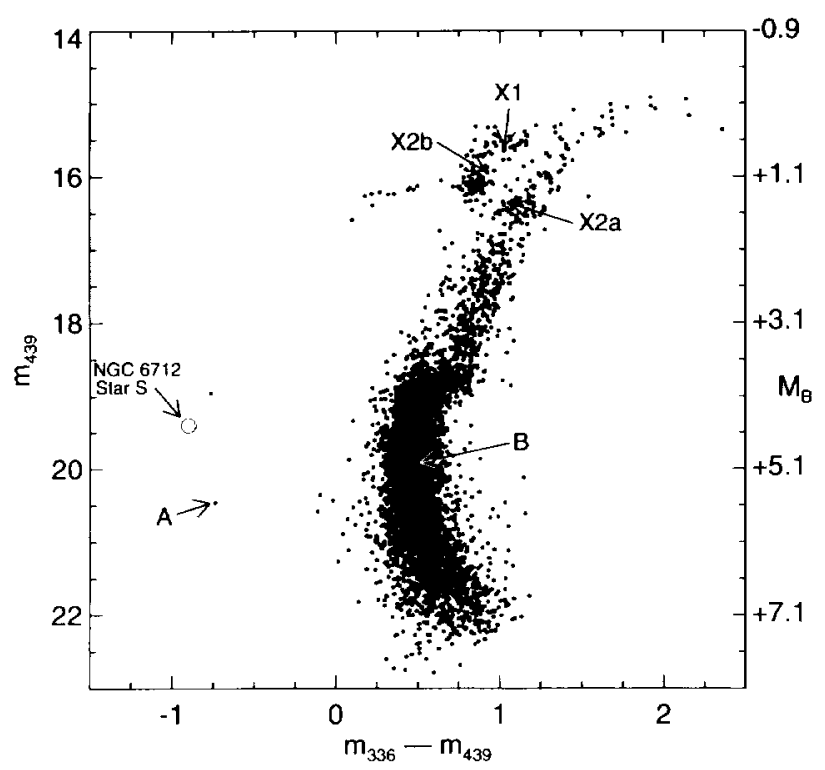

Flci. 2.-Color-magnitude diagram for $\sim 5500$ stars in the PC frame. Magnitudes are in the STMAG system; see text for details. All objects which are discussed in the text are labeled here, including star $A$, which we select as the optical counterpart due to a significant UV excess. An approximate $M_{n}$ scale is also provided, assuming $(m-M)=15.43, E(B-V)=0.02$, and $\left(B-m_{434}\right)=0.65$. This latter correction changes slightly with stellar color; (0.65 is appropriate for F type through the hottest stars, while 0.4 is more appropriate for MO stars. We also add the distance- and redalening-corrected magnitude and color of star $S$ in NC 6712 for comparison.

our optical position for the X-ray coordinates; this is well within the $90 \%$ confidence error circle of the X-ray coordinates. We denote this object as star $A$ and provide magnitude measurements in Table l. Figure 3 (Plate L11) shows a $3^{\prime \prime} \times 3^{\prime \prime}$ region of our $P C$ data centered on star $A$. We also label star $B$, which is probably unrelated to, but blended with, star A, at a separation of only 0.12 . The photometry of these objects is slightly hampered by the fact that the elongation of PSFs, discussed in $\$ 2.1$, is in nearly the same direction as the orientation of these two stars. Future observations will be complicated by the proximity of the two objects.

We have also examined the data for possible time variability of Star A. The first and last F336W exposures are separated by $3 \mathrm{hr}$, while the first and last F439W exposure are separated by only 70 minutes. Neither series shows any significant evidence for variability, although these data do not allow an adequate study. The 1 o photometric uncertainty from photon counting statisties is only $2 \%$ and $3 \%$ for star $\mathrm{A}$ for the $\mathrm{F} 336 \mathrm{~W}$ and F439W filter exposures, respectively. However, since star A is blended with star $B$, the measurements have a higher uncertainty. On the final F336W exposure, star A was contaminated with a cosmic-ray event, increasing the error of the measurement for that exposure. We set a variability upper limit of 0.10 mag during the time of our observations. These data could not have detected any small-amplitude, short-term variability of the scale reported by Homer et al. (1996) for the globular cluster X-ray source counterpart star S in NGC 6712. Archival WF/PC images of NGC 1851 have also been examined for variability. Star $A$ is above the detection threshold only in F336W exposures taken on 1993 August 21. There is no obvious evidence that star $\mathrm{A}$ has changed in brightness during this $2.6 \mathrm{yr}$ interval, but the variability upper limit is not very stringent, $\sim 0.4$ mag.
TABLE 1

Photometry and CoOrdinates for Sele(ted) Obje(ts in NGC 1851

\begin{tabular}{|c|c|c|c|c|c|}
\hline Object & $\begin{array}{c}\alpha(20(N)) \\
5^{n} 14^{m} \\
(s)\end{array}$ & $\begin{array}{l}\delta(2000) \\
-40002^{\prime} \\
\text { (arcscc) }\end{array}$ & $m_{3,30}$ & $m_{4,34}$ & $\left(m_{3,36}-m_{4,39}\right)$ \\
\hline Star $A^{a} \ldots$. & 6.428 & 38.38 & 19.73 & 20.46 & -0.7 .3 \\
\hline Star B ...... & 6.425 & 38.26 & 20.43 & 19.92 & 0.51 \\
\hline Star $X 1^{b} \ldots \ldots \ldots$ & 6.523 & 35.81 & 16.58 & 1.5 .52 & 1.06 \\
\hline Star $X 2 a^{\circ} \ldots \ldots$ & 6.556 & 36.81 & 17.51 & 16.40 & 1.11 \\
\hline Star $\times 2 b^{6} \ldots \ldots$ & 6.592 & 36.59 & 16.85 & 15.92 & 0.93 \\
\hline
\end{tabular}

"Optical counterpart for X0512-401 suggested in this work.

besignation from Aurière el al. 1994.

' X2, a designation from Aurière et al. 1994, is multiply resolved in the current work.

Since star A is not the only UV-excess object in its part of the CMD, there is some probability that it is not the X-ray counterpart but rather an unrelated object similar to the other cluster UV objects. To estimate the probability, we measure magnitudes for all stars in the PC field as well as the three WF fields. Although this larger sample has 13 times more area, it contains only 3 times more stars than the PC field alone, as the latter is centered near the cluster core and there is a sharp radial gradient of stellar density. Of the 16,000 stars for which we obtain good measurements, we find four objects with significant UV excess of $\left(m_{3,36}-m_{434}\right) \leq-0.5$ in the range $15 \leq m_{434} \leq 22$. We also search each of the fields with an image-subtraction technique to further ensure that no UVexcess objects are missed.

Therefore, approximately $2.5 \times 10^{4}$ stars with $m_{434}<22$ in the cluster are markedly UV excess, and as we measure 194 stars within the error circle, we estimate a $\sim 5 \%$ probability that one of these UV-excess objects unrelated to the X-ray source will be found in the error circle by chance. The hazards of these a posteriori probability estimates are well known, so this result must surely be regarded as qualitative rather than quantitative. The nature of the faint UV-cxcess objects observed by $H S T$ in other globular cluster cores has been recently discussed by numerous authors.

In NGC 6712 the markedly UV-excess optical counterpart star $S$ is surprisingly optically subluminous as compared with LMXRBs in the ficld (Anderson et al. 1993). If the magnitude and color of star $S$ reported in Homer et al. (1996) are corrected for the difference in reddening and distance 2 between NGC 6712 and NGC 1851, one estimates that star S, were it present in NGC 1851, would have $m_{4,34}=19.4$, $\left(m_{336}-m_{439}\right)=-0.9$ in this CMD. Therefore, while the colors of star $S$ and the candidate presented here are similar, star $\mathrm{S}$ is more luminous by $1 \mathrm{mag}$. If star $\mathrm{A}$ is the correct identification of the NGC $1851 \mathrm{X}$-ray source, the luminosity discrepancy of this subclass of objects from the field LMXRBs is further exacerbated.

To quantify this issue, we use the STSDAS SYNPHOT package and find that the blackbody spectrum which best fits the star A color $\left(m_{3,3,}-m_{4,4}\right)=-0.73$ has $T \sim 20,000 \mathrm{~K}$. Using a thermal spectrum of this temperature, we derive approximate relations to convert to Johnson $U$ and $B$ magnitudes: $\left(U-\mathrm{m}_{33}\right)=0.43$ and $\left(B-\mathrm{m}_{434}\right)=0.65$. Applying these zero-point offsets, we estimate $U=20.2, B=21.1$, and $(U-B)=-0.9$. A typical LMXRB in the field has

$2 A$ distance of $12.2 \mathrm{kpc}$ and $E(B-1)=0.12$ for NGC 1851 , and $6.8 \mathrm{kpc}$ and $E(B-V)=0.46$ for NGC 6712. were adopted from Peterson (1903). The reddening curve of Savage $\&$ Mathis $(1979)$ was used. 
$M_{V} \sim 1.2,(B-V) \sim 0$, and $(U-B) \sim-0.9$ (van Paradijs 1983). Therefore, the NGC 1851 candidate has $(U-B)$ typical of LMXRBs but is underluminous by over $4 \mathrm{mag}$, with $M_{B}=5.6$. One model for the system described by Callanan et al. (1995) invokes a subgiant secondary star; our faint counterpart rules out this possibility.

\section{CONCLUSION}

We recompute and confirm the previously published optical position of the Einstein HRI $3^{\prime \prime}$ radius error circle for the bright X-ray source in NGC 1851 using the HST GSC as a reference frame. With WFPC2 observations, 194 stars are resolved and photometered within the error circle. Star X1, the best candidate previously offered in the literature, is resolved as a single star, but is not remarkable in a CMD of the region. Star X2 is resolved as two separate stars, the brighter of which is more UV-excess than X1 but still is not remarkable.

However, we find two UV-excess objects in the PC frame, one of which is only 2 ". 15 from the X-ray source position. Therefore, we suggest this as a much more likely candidate for the optical counterpart of the X-ray source, and we denote it as star A. We search for variability, and find none, for this object in these and archival data. However, the time coverage is poor, and a proper variability study is desirable. We find a total of four markedly UV-excess objects in the $\sim 16,000$ objects measured in all four WFPC2 fields, and therefore estimate that there is $\sim 5 \%$ probability that a UV-excess star has fallen in the Einstein HRI $3^{\prime \prime}$ radius error circle by chance. Future studies of star A will be complicated by the presence of an unremarkable but comparably bright companion 0 ". 12 distant.

Star S in NGC 6712 is known to be significantly underluminous in the optical compared to the typical LMXRBs in the field. Star A in NGC 1851 is yet 1 magnitude fainter. It will be interesting to learn whether we are merely sampling a broad underlying luminosity dispersion or are instead discerning a consequence of a physical property of cluster LMXRBs that is fundamentally different from those in the field.

We are grateful to Holland Ford for supplying a groundbased CCD image of NGC 1851, to Kerry McQuade for extracting the GSSS image from the ST Scl archives, and to Ralph Bohlin, George Djorgovski, and collaborators for amicable sharing of $H S T$ data. Financial support for this work was provided by NASA through grant NAG5-1630.

\section{REFERENCES}

Anderson, S. F., Margon, B., Deutsch, E. W., \& Downes, R. A. 1993, AJ, 106, 1049

Aurière, M., Bonnet-Bidaud, J. M., \& Koch-Miramond, L. 1994, A\&A, 284, 457 (ABK)

Aurière, M.. \& Koch-Miramond, L. 1992, A\&A, 263, 82

Aurière, M., Le Fèvrc, O., \& Terzan, A. 1984, A\&A, 138, 415

Bailyn, C. D., Cool, A., Grindlay, J. E., Cohn, H., Lugger, P. M., \& McClure, R. D. 1991, in ASP Conf. Ser. 13, The Formation and Evolution of Star Clusters, ed. K. Janes (San Francisco: ASP), 363

Callanan, P. J., Penny, A. J., \& Charles, P. A. 1995, MNRAS, 273, 201

Charles, P. A., Jones, D. C., \& Naylor, T. 1986, Nature, 323, 417

Clark, G. W. 1975, ApJ, 199, L143

Clark, G. W., Markert, T. H., \& Li, F. 1975, ApJ, 199, L93

Cudworth, K. M. 1988, AJ, 96,105

Deutsch, E. W., Anderson, S. F., Margon, B., \& Downes, R. A. 1994, BAAS, 26, 1488

Downes, R. A., Anderson, S. F., \& Margon, B. 1996, PASP, 108, 688

Forman. W., \& Jones, C. 1976, ApJ, 207 , L177

Grindlay, J. E., Hertz, P., Steiner, J. E., Murray, S. S., \& Lightman, A. P. 1984, ApJ, 282, Li3

Holtzman, J. A., Burrows, C. J., Casertano, S., Hester, J. J., Trauger, J. T.,

Watson, A. M., \& Worthey, G. 1995 a, PASP, 107, 1065

Holtzman, J. A., et al. 1995b, PASP, 107, 156
Homer, L., Charles, P. A., Naylor, T., van Paradijs, J., Aurière, M., \& Koch-Miramond, L. 1996, MNRAS, in press

Katz, J. 1. 1975, Nature, 253, 698

King, I. R., et al. 1993, ApJ, 413, L117

Landsman, W. B. 1993, in ASP Conf. Ser. 52, Astronomical Data Analysis Software and Systems II, ed. R. J. Hanisch, R. J. V. Bissenden, \& J. Barnes (San Francisco: ASP), 256

Lasker, B. M., Sturch, C. R., McLean, B. J., Russell, J. L., Jenkner, H., \& Shara, M. M. 1990, AJ, 99, 2019

Margon, B., Anderson, S. F., Downes, R. A., \& Bohlin, R. C. 1992, in Science with the Hubble Space Telescope, ed. P. Benvenuti \& E. Schreier (Garching: ESO), 421

Nieto, J.-L., et al 1990, A\&A, 239, 155

Peterson, C. J. 1993, in ASP Conf Ser 50 Structure and Dynamics of Globular Clusters, ed. S. G. Djorgovski \& G. Meylan (San Francisco: ASP), 337

Russell, J. L., Lasker, B. M., McLean, B. J., Sturch, C. R., \& Jenkner H. 1990, AJ, 99,2059

Saha, A., Sandage, A., Labhardt, L., Tammann, G. A., Macchetto, F. D., \& Panagia, N. 1996 , ApJ, 466,55

Savage, B. D. \& Mathis, J. S. 1979, ARA\&A. 17.73

Schechter, P., Mateo, M., \& Saha, A. 1993, PASP, 105, 1342

van Paradijs, J. 1983, in Accretion Driven X-ray Sources, ed. W. Lewin \& E. van den Heuvel (Cambridge: Cambridge Univ.), 189 


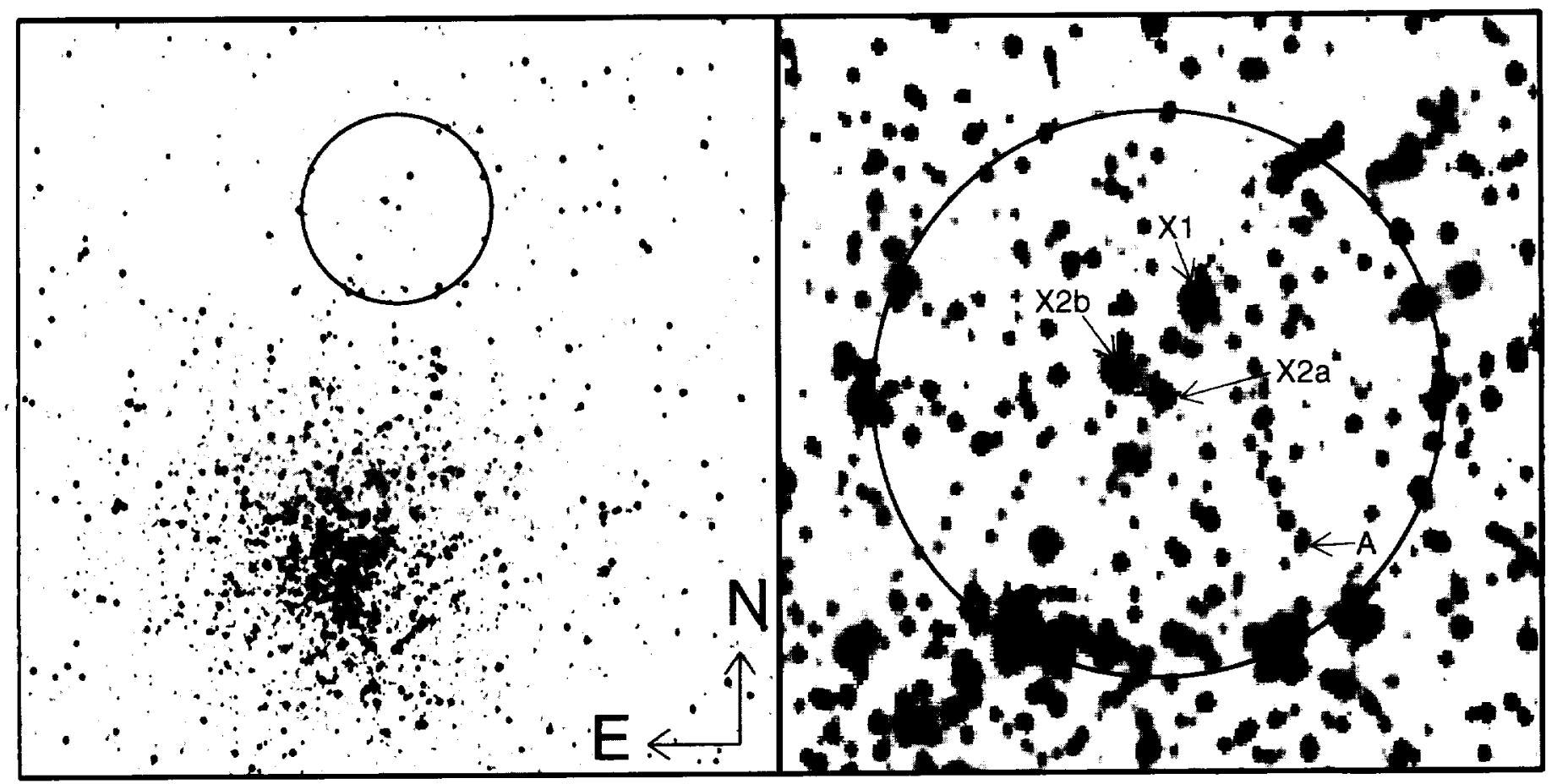

Fik. 1.-Left pant: $24^{\prime \prime} \times 24^{\prime \prime}$ section of the HST PC image of NGC 1851 with the F336W filter. The image has been reoriented so that north is up and east is to the left. A 3" radius error circle is drawn about the position of the X-ray coordinates. Right panel: $8^{\prime \prime} \times 8^{\prime \prime}$ section of the same image centered at the X-ray coordinates. A cross denotes the optieal position, derived in this paper from the GSC source data, of the X-ray coordinates, and the $3^{\prime \prime}$ radius error circle is overlaid. Several ohjects discussed in the text are labeled. Star $A$ is selected as the optical counterpart due to a significant UV excess.

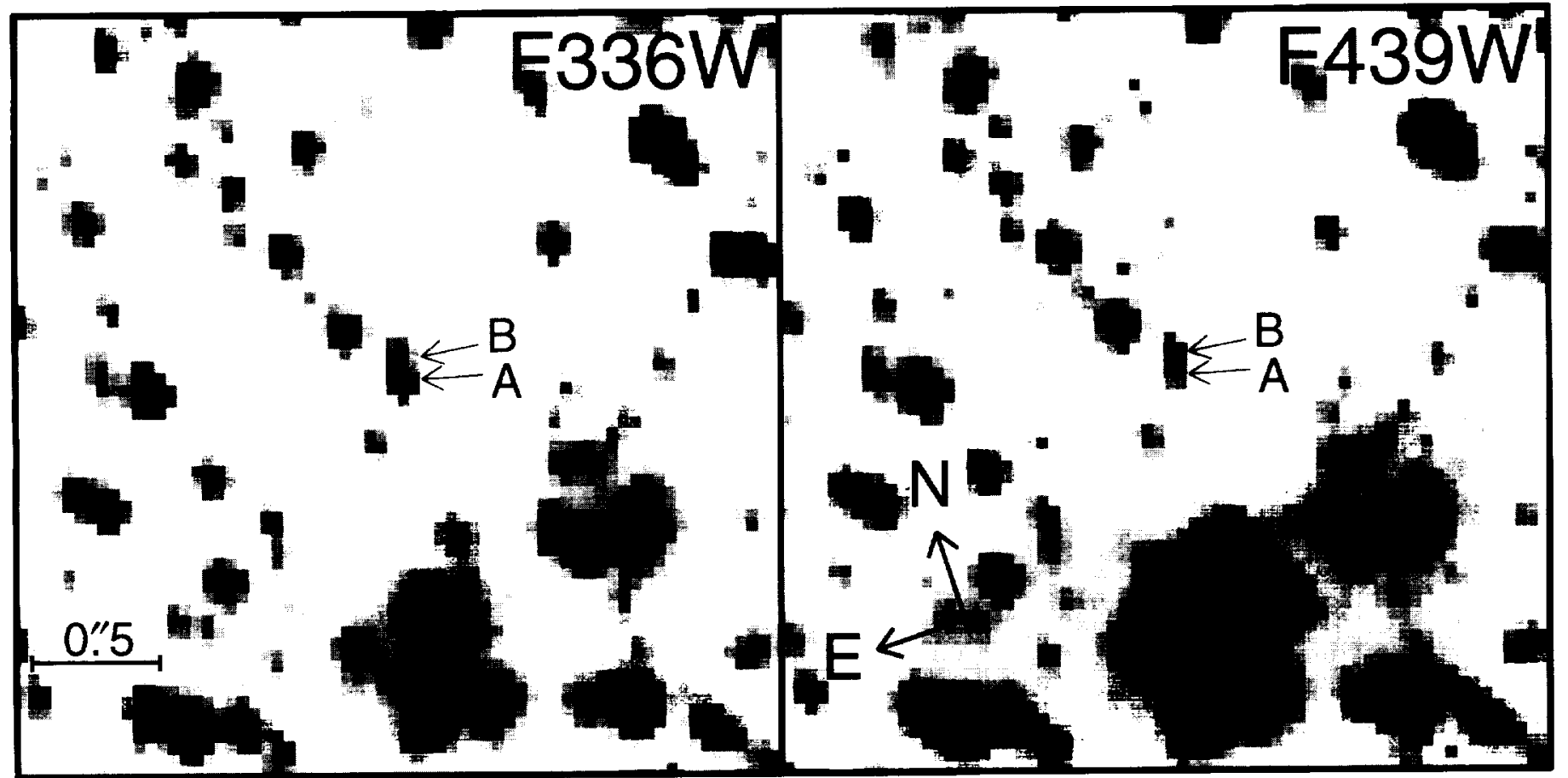

FII. 3- $3^{\prime \prime} \times 3^{\prime \prime}$ sections of the F336W $(U)$ and F439W $(B)$ images centered on star A, which we select as the optical counterpart due to at significant UV excess. These images are still in the original orientation, so north and east are labeled. Star B, 0.12 distant, is apparently unremarkable. hut may complicate future abservations. 
